\section{Cancer management during COVID-19 period-A preliminary study}

\author{
Anita Ramesh', Praveen Sahu' ${ }^{2}$, Lakshya Mittal ${ }^{2}$, \\ Raji Sundararajan ${ }^{2 \star}$, Ignacio Camarillo ${ }^{3}$
}

Address for correspondence: Raji Sundararajan, School of Engineering Technology, Purdue University, West Lafayette, IN 47907-USA. Email: raji@purdue.edu

How to cite this article: Ramesh A, Sahu P, Mittal L, Sundararajan R, Camarillo I. Cancer management during COVID-19 period-A preliminary study. G Med Sci. 2020; 1(6): 053-068.

https://www.doi.org/10.46766/thegms.oncol.20122003

Copyright: (c) 2020 Anita Ramesh, Praveen Sahu, Lakshya Mittal, Raji Sundararajan, Ignacio Camarillo. This is an Open Access article distributed under the Creative Commons Attribution License, which permits unrestricted use, distribution, and reproduction in any medium, provided the original work is properly cited.

${ }^{1}$ Saveetha Medical College \& Hospital, Chennai, India

${ }^{2}$ School of Engineering Technology, Purdue University, West Lafayette, IN 47907USA

${ }^{3}$ Biological Sciences Department, Purdue University, West Lafayette, IN 47907-USA

\begin{abstract}
The ongoing COVID-19 pandemic has now thrown us into turmoil by adversely affecting the entire socio-economic structure of the world. Currently (Dec $20,2020)$, there are 76.4 million incidences worldwide and 17.6 million in the USA, and counting. Primarily in this scenario, all the resources available at hospitals and medical care units are reasonably redirected to address the contagious COVID-19 infected patients. This is an issue for a cancer patient, who may be at risk or infected with Coronavirus needs to be managed amid multiple unknown complications. Many issues are still unanswered and an attempt to streamline various strategies is being offered by Oncologists around the world to treat cancer patients. The gravest concerns are related to deciding suitable supervision for a positive COVID-19 cancer patient undergoing a cycle of chemo/radiotherapy; handling advanced stage of cancer in regions profoundly affected by virus; evaluating the risk-benefit parameters for treatment or surgeries; planning routine screening and precaution for weak patients, medical staff and others in the community. Here we have presented the different guidelines, schemes, and strategies accessible from several Oncology departments and organizations in multiple countries.
\end{abstract}

\section{Introduction}

The cancer patients undergoing chemo or radiation therapy or done with surgery, may have weakened or compromised immune systems [1][2][3], and hence will be more vulnerable to infections, such as COVID-19 infection. For instance, anesthesia given during surgery will take days to weeks to get back their original immune capacity. Similarly, chemotherapy can weaken the immune system due to the chemo-drugs, which will affect not only the cancer cells but also normal cells and further decrease the number of white blood cells in the body. Radiation can also cause low white blood cells, which increases the risk of infections. Besides, chemo and radiation therapy patients will lose their normal fighting capacity because of loss of nutrition caused by nausea, vomiting, loss of appetite, unable to eat due to pain, and various other reasons. Accordingly, the American Society of Oncology (ASCO) [4], the European Society for Medical Oncology ESMO [5], and all other agencies are providing guidelines to be adopted during this ongoing pandemic. For example, American Cancer Society guidelines indicate that for cancer patients during the COVID-19 pandemic, screening schedules should be delayed [6].

Furthermore, the casualty of the cancer patients in a New York Hospital System reported by Mehta et al. in a May 1st publication [7] indicates that the death rate of COVID-19 cancer patients was double that of regular COVID-19 patients and confirming at least in this case 
that cancer patients are a high-risk group considering the continuing pandemic. Such patients are already exposed to infection due to the underlying illness and possible immuno-suppressed state; hence they are at increased risk for developing complications from the viruses. Therefore for those with COVID-19, the treatment of the aforementioned takes priority over cancer treatment that can be delayed. However, it has to be done on a case-bycase basis for each patient. Also, patients in quarantined cities who are unable to travel for appointments or facing difficulties in procuring the required medications, the risk of interruptions in drug supply chains, and consequent shortages will exacerbate this issue. Additionally, scheduled operations, non-urgent cancer treatments could be canceled or postponed to address the pandemic issue primarily. Oncological resources and personnel would be redirected for the pandemic treatment. Cancer patients must bear this also, in addition to their high-risk and susceptibility to the pandemic. Reviewed in this article are the various guidelines and real-world experiences of various hospitals [2-27].

\section{Cancer management guidelines at Chennai, India [8], [9]}

Even though cancer services may not seem to be in the frontline as the existing planning and resources are devoted to addressing the coronavirus pandemic issue yet oncologists do have a crucial role to play. They explore and act upon various national and local guidelines meant for the management of Cancer patients during such unforeseen circumstances. In view of the same, we will discuss in the forthcoming sections various factors placing cancer patients at higher risk and the list of recommendations and guidelines prescribed during the current state.

\section{Factors elevating the risk of COVID-19 in cancer patient}

According to medical oncology specialists the more numerous of these singular factors a cancer patient has, the more prone they are to catch a severe illness with coronavirus particularly if treated with systemic anti-cancer therapies. These factors are described in Figure 1 for a comprehensive understanding. The cancer patients undergoing active chemotherapy, radiotherapy, immunotherapy, or other continuing antibody treatments for cancer, or targeted cancer treatments might develop pneumonitis or other related symptoms, which can further put them in conditions whereby coronavirus infection could be worse. Similarly, patients having blood or bone marrow cancer, such as leukemia, lymphoma, and myeloma; recent history of bone marrow/stem cell transplants in the last 6 months are more susceptible. In addition to immunosuppression, diverse factors related to comorbidities such as elderly age ( $>60$ yrs), pre-existing cardiovascular \& respiratory ailments need special care.

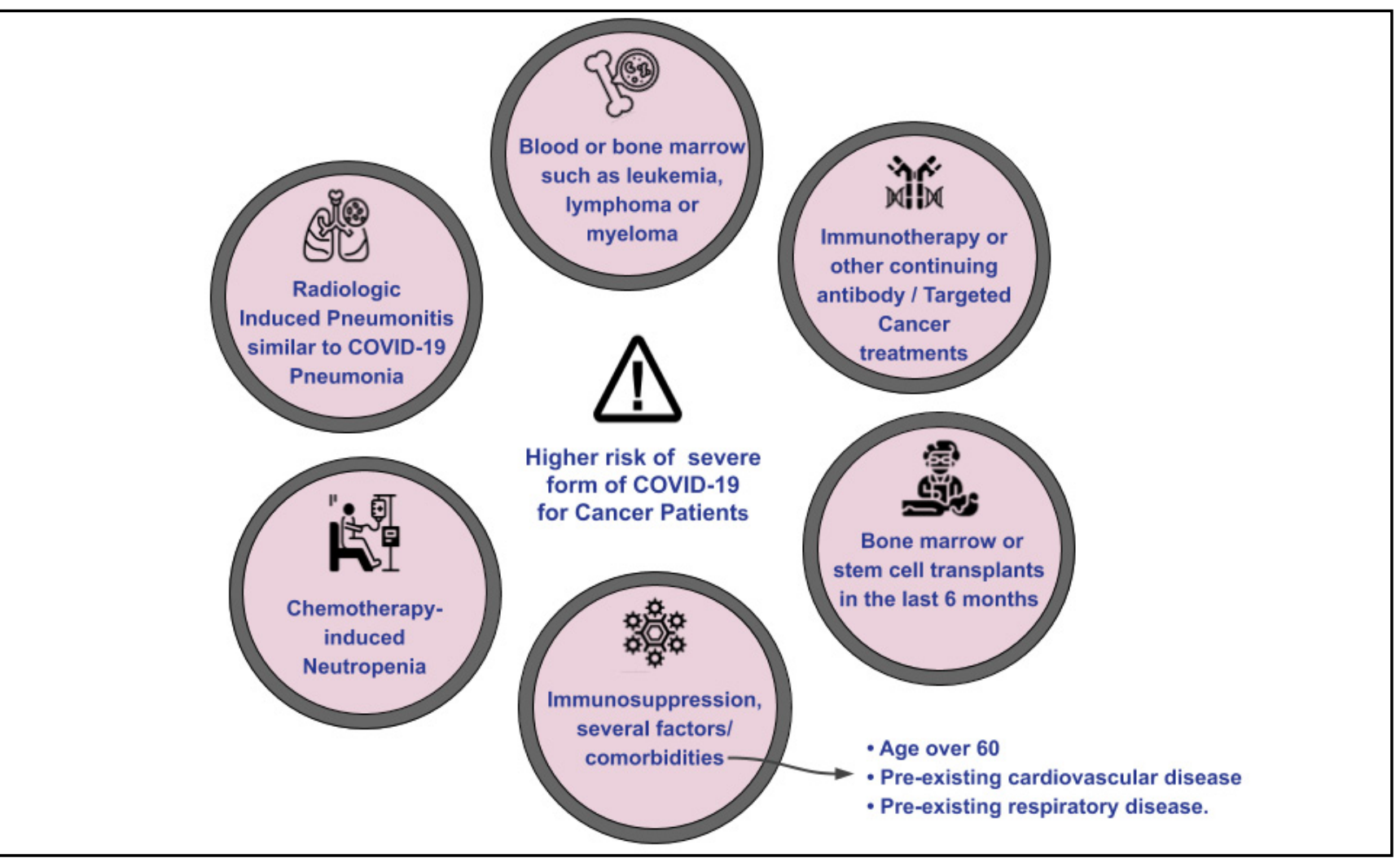

Figure 1. Multiple factors that can put a Cancer patient at higher risk of COVID-19 infection 


\section{New guidelines and recommendations [10]}

In the aforementioned cases, clinicians may also need to prioritize treatment for those most in need. But, the decisions taken are done with the multidisciplinary team (MDT) input and communicated with patients about various alternatives which will be covered in this section. It requires careful categorization of patients and setting up priority levels based on the urgency on a case by case basis by intent and risk-benefit ratio associated with treatment. For instance, some of the life-threatening cases including bleeding, localized infection, and permanent injury from the progression of spinal cord compression require immediate attention.

Additionally, clinicians need to weigh the level of immunosuppression linked with personalized therapy and patients' other risk factors while categorization of patients. This will also vary according to the type of tumor, whereas the general approach is to prioritizing patients on systemic anti-cancer therapy. Besides, it is always advised considering alternative and less resourceintensive treatment regimes.

\section{Home is the new hospital}

Patients should opt for home care whenever it is medically and logistically feasible for the medical team and caregivers. Those who are not on active cancer treatment should avoid regular visits to doctors for routine followups.

Hospital visits for palliative therapy can be postponed unless it is an emergency. For patients with fever or other symptoms of infection, routine lab samples can be collected from home, instead of visiting the clinic.

\section{Embrace technology}

This is the appropriate time to make extensive use of telemedicine for consultations and patient-doctor interactions. Patients who are on follow-up, oral drugs, or maintenance therapy in remission can consult their oncologists via Mobile Phone, WhatsApp, or Email, and avoid unnecessary hospital visits.

\section{Screen patients, donors, and visitors}

Doctors should duly screen patients for COVID-19 before the initiation of any treatments that are already being scheduled and cannot be postponed. It is better to get the screening done one day in advance, as that saves time during the cancer treatment (however that may involve an additional visit or as recommended prefer a home collection of a blood sample). Additionally, blood donors and visitors must be screened and strict visitor hours shall be maintained to reduce the infection.

\section{When to continue the treatment?}

Clinical care must be delivered to all serious or emergency cancer cases, including oncological emergencies for radiation, as done routinely. Any medication (involving prophylactic antibiotics) or procedures of preventing diseases should continue, because they may be of potential usefulness in maintaining the overall health of the patient and making them less vulnerable to potential COVID-19 complications.

\section{When to postpone the treatment?}

For cancer patients diagnosed with COVID-19, it is preferable to postpone active anti-cancer treatments. Chemotherapy can be stopped for the infected patients when cancer is in deep remission and when it is given only as maintenance therapy.

Another option is modifying treatments. Some patients could be switched from IV-based chemo to oral pills. Physicians should decide on a case-by-case basis. When delays and modifications of treatments may pose a higher risk of compromised disease control, withholding of critical cancer treatments are not preferable. However, if the absolute benefit is minimal, COVID-19 treatments get the preference.

\section{Cancer management guidelines at Tata Memorial Hospital, Mumbai, India [11]}

The Tata Memorial Hospital (TMH) is an India's largest specialist Cancer Treatment \& Research Center and is associated with the Advanced Centre for Treatment, Research and Education in Cancer (ACTREC). The center sees 70,000 new patients with cancer each year and was quick in placing the guidelines in responses to COVID-19 extending from the contemporary reflection on policy to strategy relevant to the pandemic. This takes into consideration the social distancing norm, the difficulty faced by the patients, and healthcare workers to reach the hospital amid lockdown.

A "Multi-Pronged" approach was taken by the center while deciding to proceed with providing cancer care by taking prioritization guidelines to oversee cancer treatment choices. Following these criteria, high priority was given to the patients with possibly curable diseases who are likely to derive substantial benefit from treatment, whereas care being suspended for patients treated with palliative intent, especially those foreseen to receive limited benefit from treatment as shown in Figure 2. 

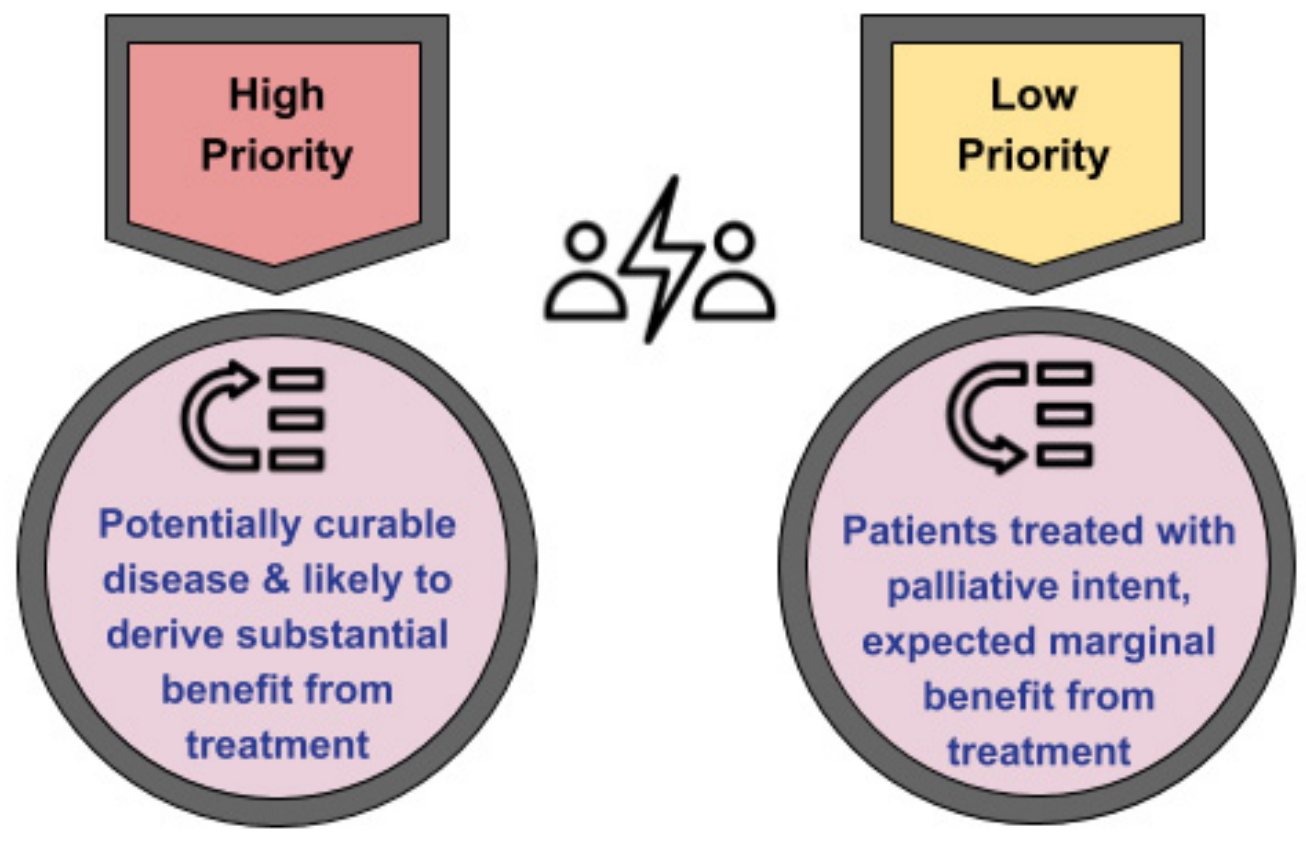

Figure 2. "Multi-Pronged" approach by TMH on prioritizing the Cancer patients

Further measures and elements of the "Multi-Pronged" policy include the following as in Figure 3.

Firstly at an administration level, a central group is formed to take action and monitor the implementation combined with every day scheduled debriefing and formulation of engagement strategies. This will immensely help in tracking the implementation and readjusting to any modification needed in an agile manner.

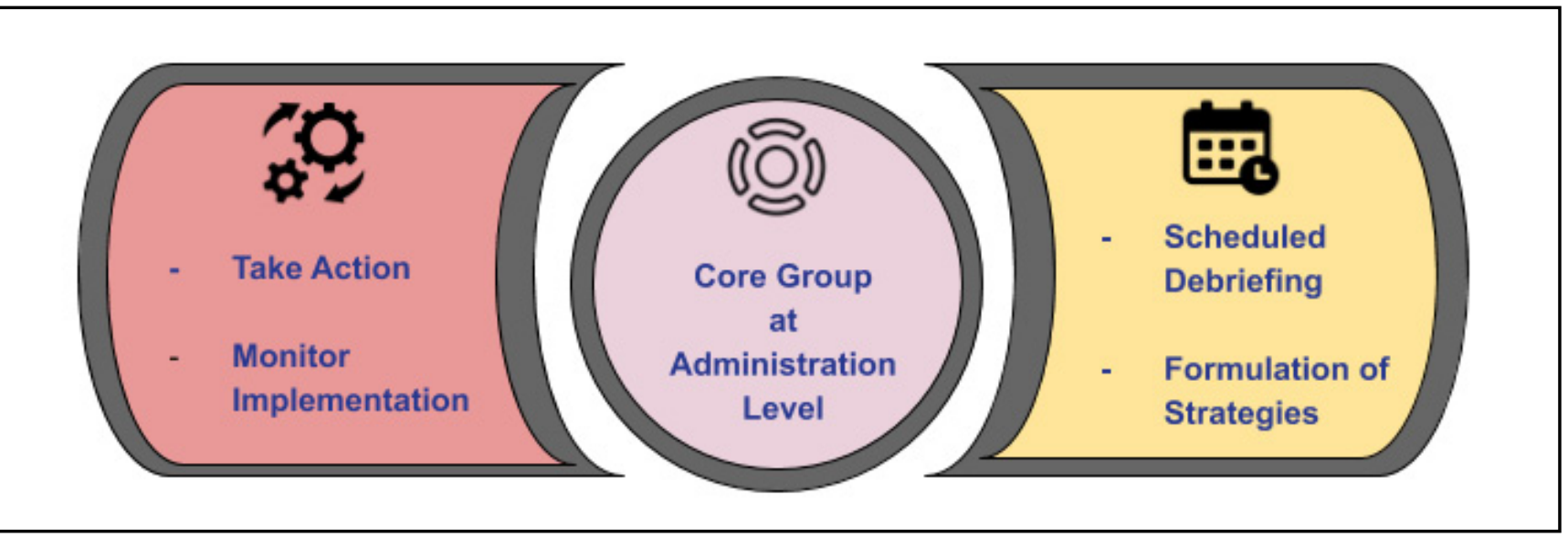

Figure 3. Administrative level "Multi-Pronged" approach by TMH

Secondly, several moves were taken towards the smooth running of the Cancer care units amid the lockdown in the country which poses a serious logistic issue. Consequently, as shown in Figure 4, complicated surgeries that might require many blood transfusions along with extended ICU-Intensive care units are being avoided. The strategy of hypofractionated where the entire dose of radiation is broken into large doses and procedures is given once a day or less often whenever viable. For instance, in the case of breast, prostate, and lung cancers and similarly the provision of palliative radiotherapy in a single portion or weekly regimens. Further, as a valid measure, the decrease in the usage of myelosuppressive systemic therapy was proposed, and wherever possible switching to oral treatment is advised. In fact, based on the degree of benefit, if marginal, the treatment can be delayed. 


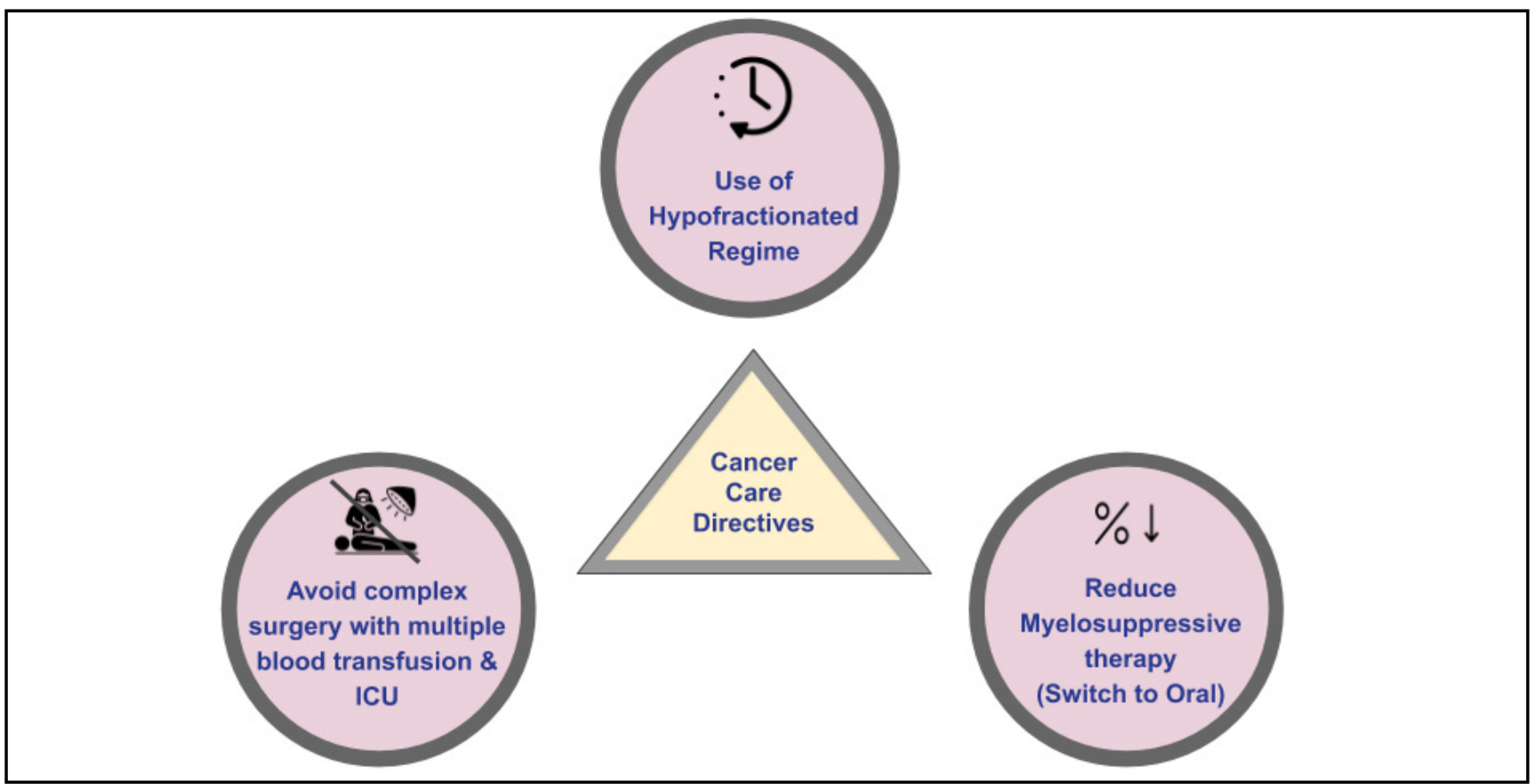

Figure 4. Cancer care directives as a part of "Multi-Pronged" approach by TMH

The next step is directed toward incoming patients even before accessing hospital premises by setting up "screening camps" to make sure that the spreading of the virus can be contained and a more certain diagnosis can be taken for COVID-19 affected Cancer patients. Additionally, by decreasing patient visits and rigorous constraint on relatives and associates in outpatient and inpatient wards by using remote counseling over phone is suggested as an alternative to routine follow-up appointments. To tackle the suspected or established COVID-19 cases the hospital developed a standard operating procedure (SOP) and used simulation drills along with a fever clinic and formation of isolation wards.

Last but not least and also perhaps the most basic is the safety of the employee, therefore certain strategies focused on granting compensated leave for high-risk staff members i.e. elderly, having various comorbidities, or who are receiving immunosuppressive medication and pregnant staff. Moreover, as an alternative possibility in case of mass quarantine, the rotation system of personnel is put in place. To achieve this, supplementary transportation means such as buses were organized for members who are not able to reach work because of ongoing lockdown.

\section{Cancer management in Wuhan, China [12]-[15]}

According to accessible statistics from Cancer Center of Wuhan Union Hospital - around 1186 Cancer patients which includes 165 patients with hematological malignancies and remained with solid tumors were admitted from Jan 15 to Feb 25, 2020. These cases are particularly challenging because the immunity of cancer patients is generally compromised in contrast to other patients. Thus, the dependency on the availability of medical support is high, making them considerably vulnerable as a consequence of the epidemic. As witnessed and depicted in Table 1 and Figure 5, six out of 13 patients with blood cancer died i.e. fatality rate of $46 \cdot 2 \%$, whereas two out of 20 patients with solid tumors died i.e. fatality rate $10.0 \%$ at the aforementioned center. In conclusion, patients with blood cancer were more prone to SARSCoV-2 infection than patients with solid tumors. It is reasoned that aggressive chemotherapeutic protocols, agranulocytosis, and impaired immunity is the cause of greater fatality rate in patients with blood cancer.

\begin{tabular}{|c|c|c|}
\hline & $\begin{array}{c}\text { Blood } \\
\text { Tumors }\end{array}$ & $\begin{array}{c}\text { Solid } \\
\text { Tumors }\end{array}$ \\
\hline $\begin{array}{c}\text { Total No. of } \\
\text { Patients }\end{array}$ & 165 & 1021 \\
\hline $\begin{array}{c}\text { Infected with SARS-CoV-2 } \\
\text { virus }\end{array}$ & 13 & 20 \\
\hline $\begin{array}{c}\text { Fatality Rate [COVID cancer } \\
\text { patients } \\
\begin{array}{c}\text { death w.r.t. No. of coronavi- } \\
\text { rus infected patients] }\end{array}\end{array}$ & $6[46.2 \%]$ & $2[10 \%]$ \\
\hline
\end{tabular}

Table 1. Blood Tumors Vs. Solid Tumors Fatality Rate 


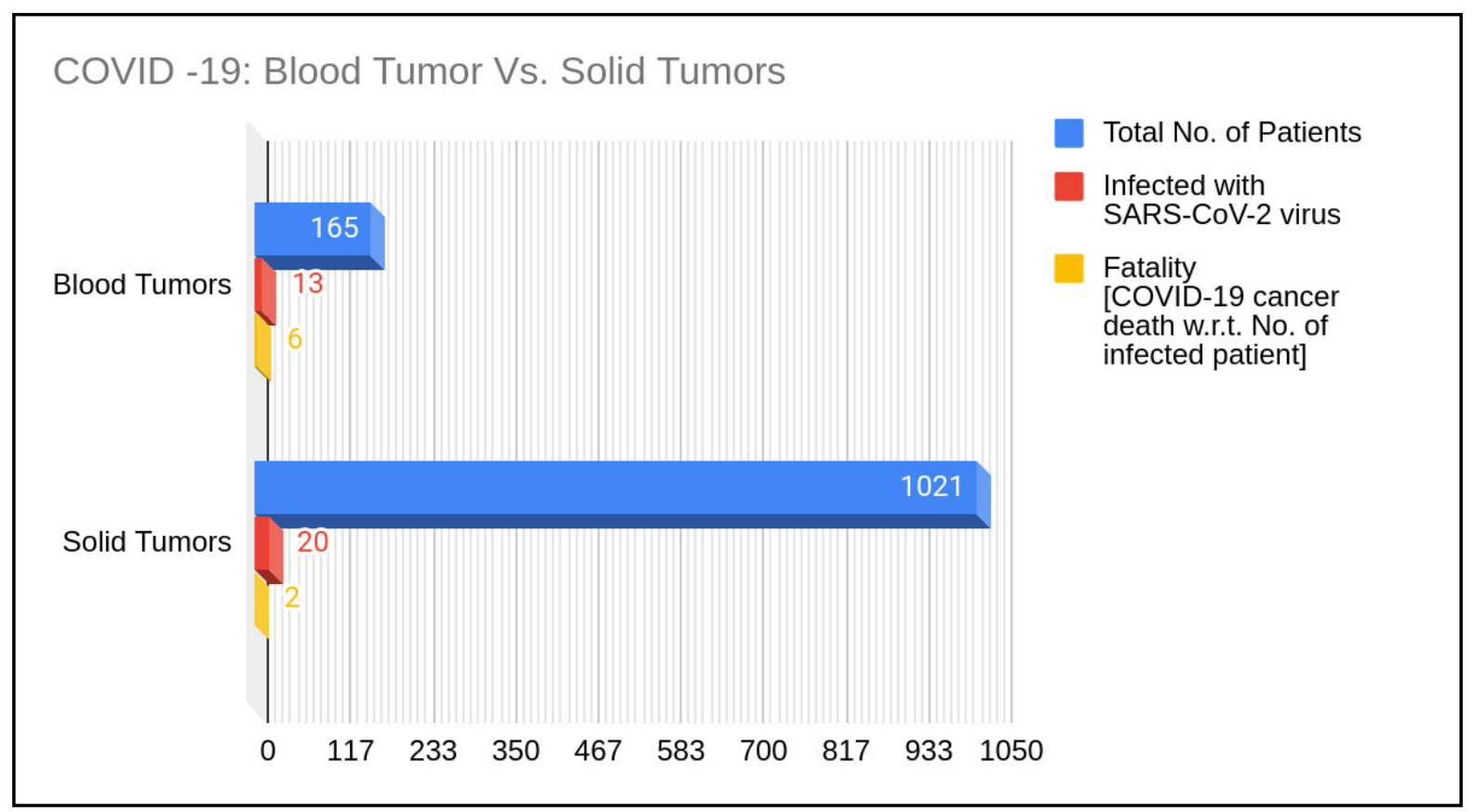

Figure 5. Analysis of COVID-19 cases with Blood Tumors and Solid Tumors

Further, it was found that 8 patients operated by targeted therapies (kinase and proteasome inhibitors) and 2 getting immune checkpoint inhibitors out of a total of 33 cancer patients with COVID-19 conferred more affirmative results than those handled with chemotherapy.

It is worth mentioning here that throughout the midst of January, due to lack of tests, the exact diagnosis of COVID-19 was not performed on admitted cancer patients who showed symptoms such as fever and diarrhea. This led to the cross-infection where the COVID-19 infected person socialized with other patients. All this was the result of a lack of knowledge concerning the new coronavirus, shortage in stock of nucleic acid testing kits, lack of protection, scarcity of beds, and poor isolation gave rise to a large number of infected.

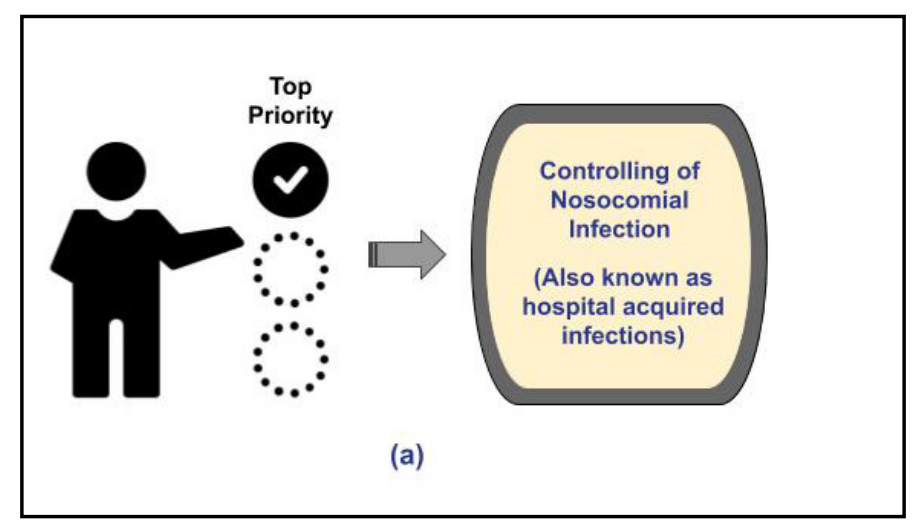

Through this learning, precautionary steps were intensified for all at early-stage testing for all including patients, caregivers, and medical personnel using nucleic acid tests, antibody tests, and CT scans. The medical staff was trained to work towards early detection, immediate isolation, and quick treatment and to efficiently shield themselves from the virus. This step helps avoid the grave shortage of staff that the hospital might have to face during such a crisis situation. Additionally, a number of doctors and nurses teams were re-deployed and tentatively assigned from other not-in-service departments to oncology departments for the smooth operation of cancer patients. A private quarantine protocol was put in place to alleviate any risk of infection for the cancer patients undergoing treatment or monitored in a hospital environment due to the immunosuppressive characteristics of chemotherapy and radiotherapy while holding nosocomial infection at top priority by management as shown in Figure 6.

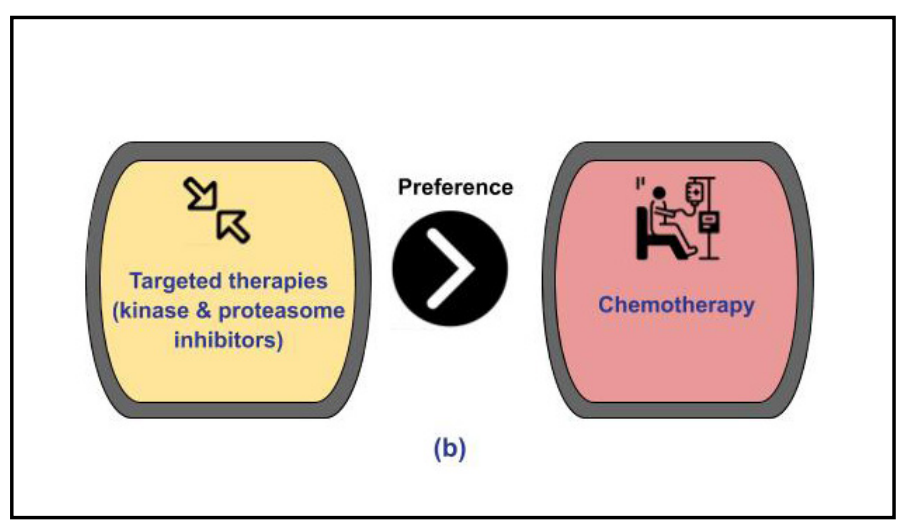

Figure 6. (a) Nosocomial infection was set as a top priority by Hospital Management; (b) Preference between Targeted therapies (Kinase \& Proteasome Inhibitors) 
Some of the mandatory rules were implemented throughout, such as wearing surgical masks, hand sanitation, and separate disposal of patient waste and demoting the number of hospital visits of cancer patients and visitors to overcome cross-contamination with who are at home or visiting online clinics. Last but not least, it is worthy to acknowledge the crucial role played by telemedicine in the diagnosis \& treatment of patients with cancer in-home care or visiting online clinics.

\section{COVID-19 death rate in cancer patients in NYC [7]}

The New York (NYC) metropolitan city alone saw above 200,000 novel coronavirus cases. At a point in time during mid-April, the confirmed cases in NYC were higher than in China and the UK. Hence, it was declared an epicenter of the COVID19 pandemic in the US. In May, NYC has attained a decline stage in terms of the number of COVID-19 cases per day and the concluding count is approaching 205011 confirmed cases; 53369 people hospitalized; 17255 confirmed deaths with a positive COVID-19 and 4705 probable deaths with no positive lab report are categorized as caused by "COVID-19" or equivalent [16].

In general, close to $12500 \mathrm{NYC}$ residents die every year due to cancer, making it the 2nd most predominant reason for death after heart disease [17]. Now, explicitly w.r.t the cancer patients with COVID-19, it was found that the fatality rate is twice that of COVID-19 patients without cancer treatment. For this, the researchers analyzed the 218 cancer patients with COVID-19 with 1,090 age- and sex-matched noncancer patients with COVID-19 treated in the Montefiore Health System between March 18 and April 8, 2020. The death rate was $28 \%(61 / 218)$ for cancer patients with COVID-19, compared to $14 \%$ (149/1090) for noncancer patients with COVID-19. The mortality rate was $37 \%(20 / 54)$ for hematologic malignancies, $25 \%(41 / 164)$ for solid tumor cases, and 55\% (6/11) for lung cancer patients. Further out of 218 identified cancer patients treated for COVID-19 - 75\% of patients had solid tumors and the rest had hematologic malignancies. This data are from the Montefiore Health System between March 18 and April 8, 2020, and shown in Figure 7.

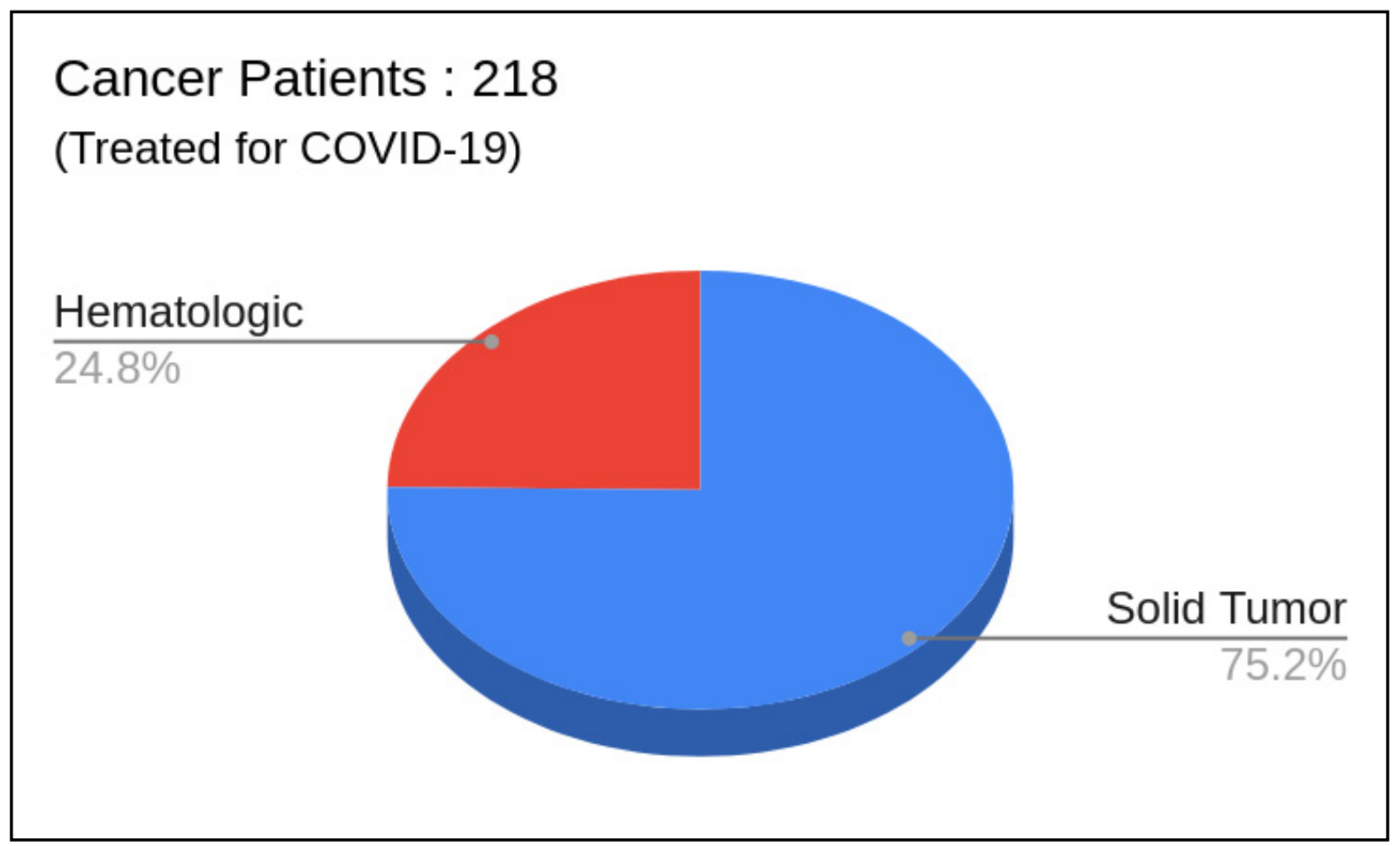

Figure 7. Comparison of the cancer patient with COVID-19: Solid Tumor vs. Hematologic cases

Overall, 61 out of 218 cancer patients died from COVID-19, including 41 out of 164 with solid tumors and 20 out of 54 of those with hematologic malignancies as depicted in Figure 8. 


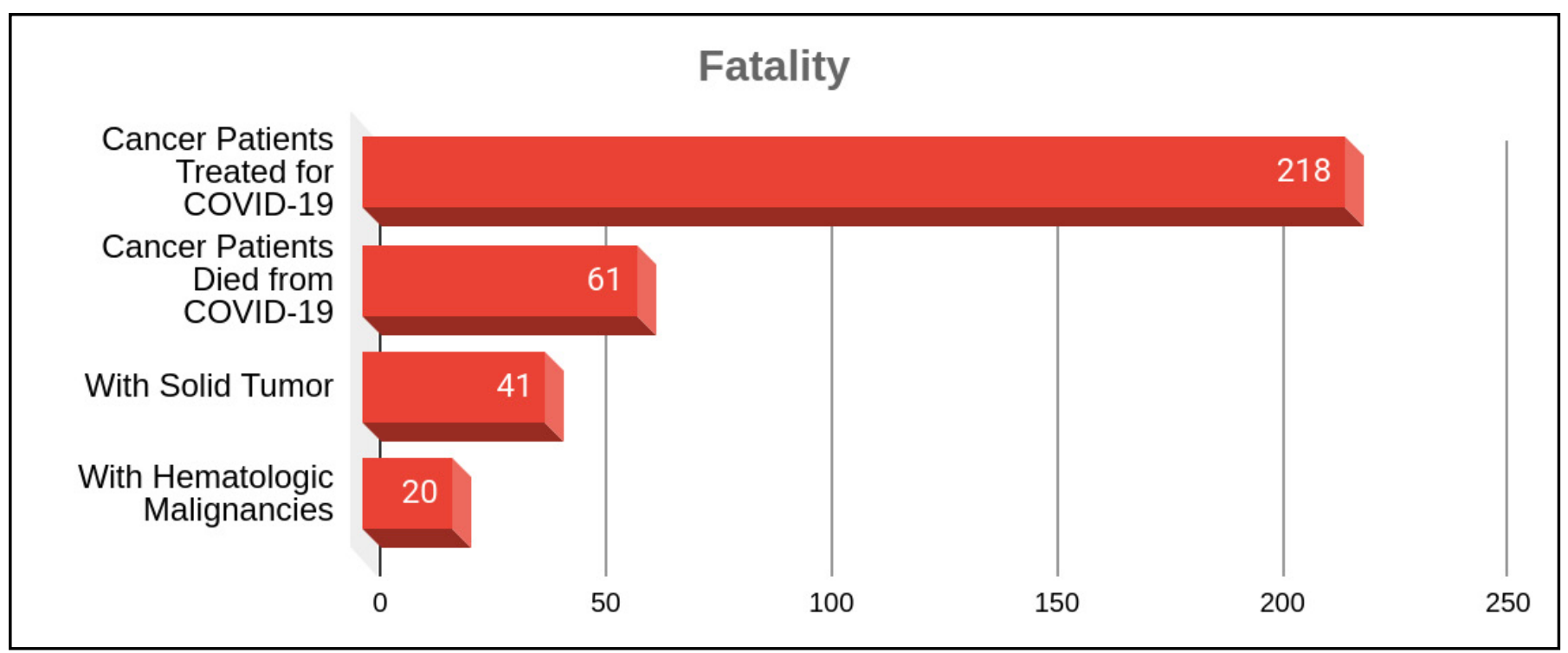

Figure 8: Comparison of fatality in the cancer patient with COVID-19: Solid Tumor vs. Hematologic

\section{Deaths by cancer type}

The casualty in solid tumor cases with COVID-19 was distributed among various cancer types, such as Pancreatic, Lung, Colorectal, Upper Gastrointestinal, Gynecologic, Skin, Hepatobiliary, Bone/Soft tissue, Genitourinary, Breast, Neurologic, and Head and Neck. The total number of cases was the highest in Genitourinary and Breast, but the casualty was confined to $15 \%$ in both cases, whereas pancreatic and lung cancer patients see more than $50 \%$ casualty, and Colorectal, Upper Gastrointestinal, Gynecologic marked more than 35 \% loss respectively, as shown Figure 9. Similarly, in the case of Hematologic Malignancies, 54 patients were spread among cancer types including Chronic myeloid leukemia, Hodgkin lymphoma, Myelodysplastic syndromes, Multiple myeloma, Non-Hodgkin lymphoma, Chronic lymphocytic leukemia, Myeloproliferative neoplasms, Acute lymphoblastic leukemia, and Acute myeloid leukemia.

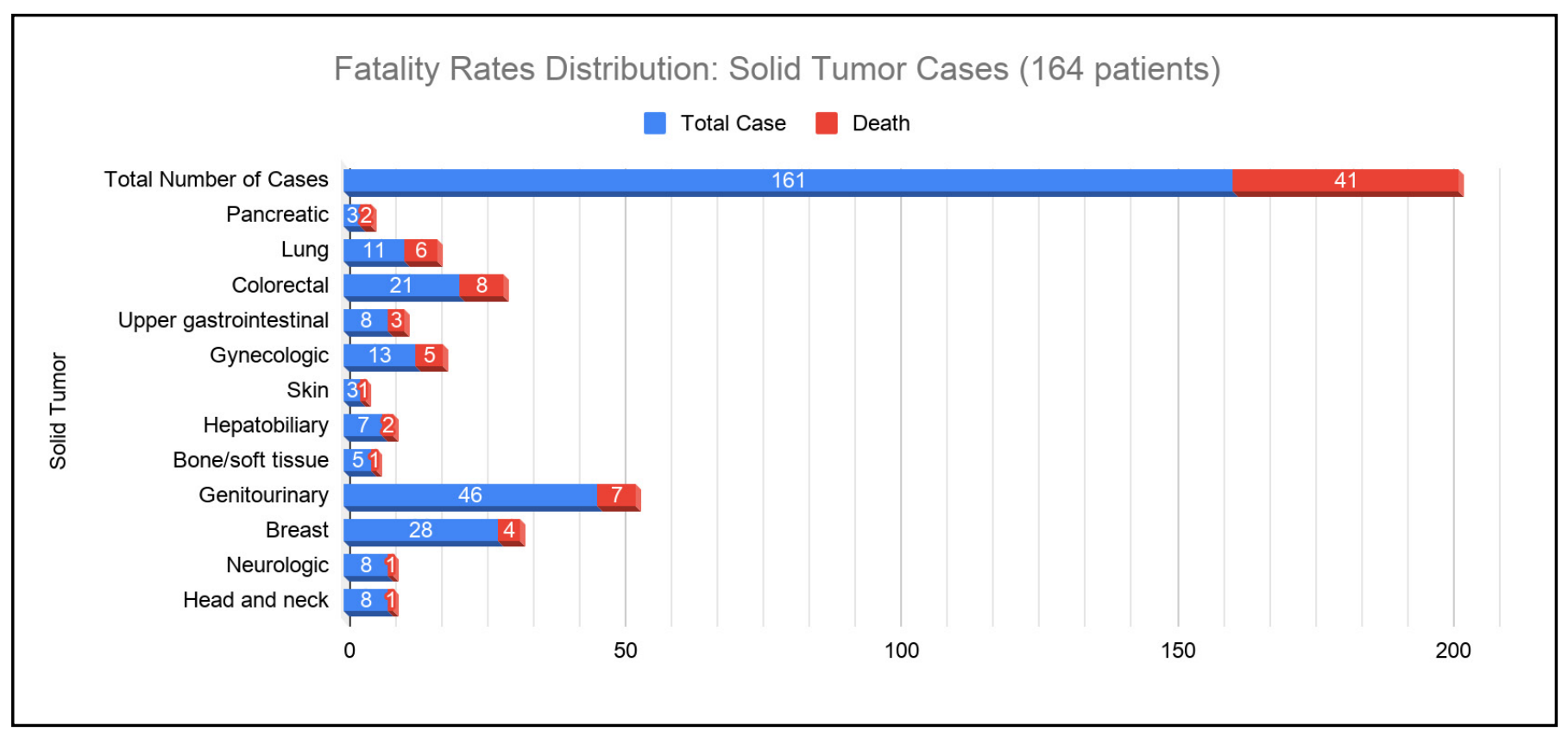

\section{Figure 9. Fatality distribution amongst the Solid tumor cancer patient with COVID-19}

Also, none of the four patients with acute lymphoblastic leukemia died, and there was one patient with acute myeloid leukemia who survived. Most of the cases were from Multiple myeloma and Non-Hodgkin lymphoma and the casualty was about $38 \%$ and 33\% each as Figure 10. 


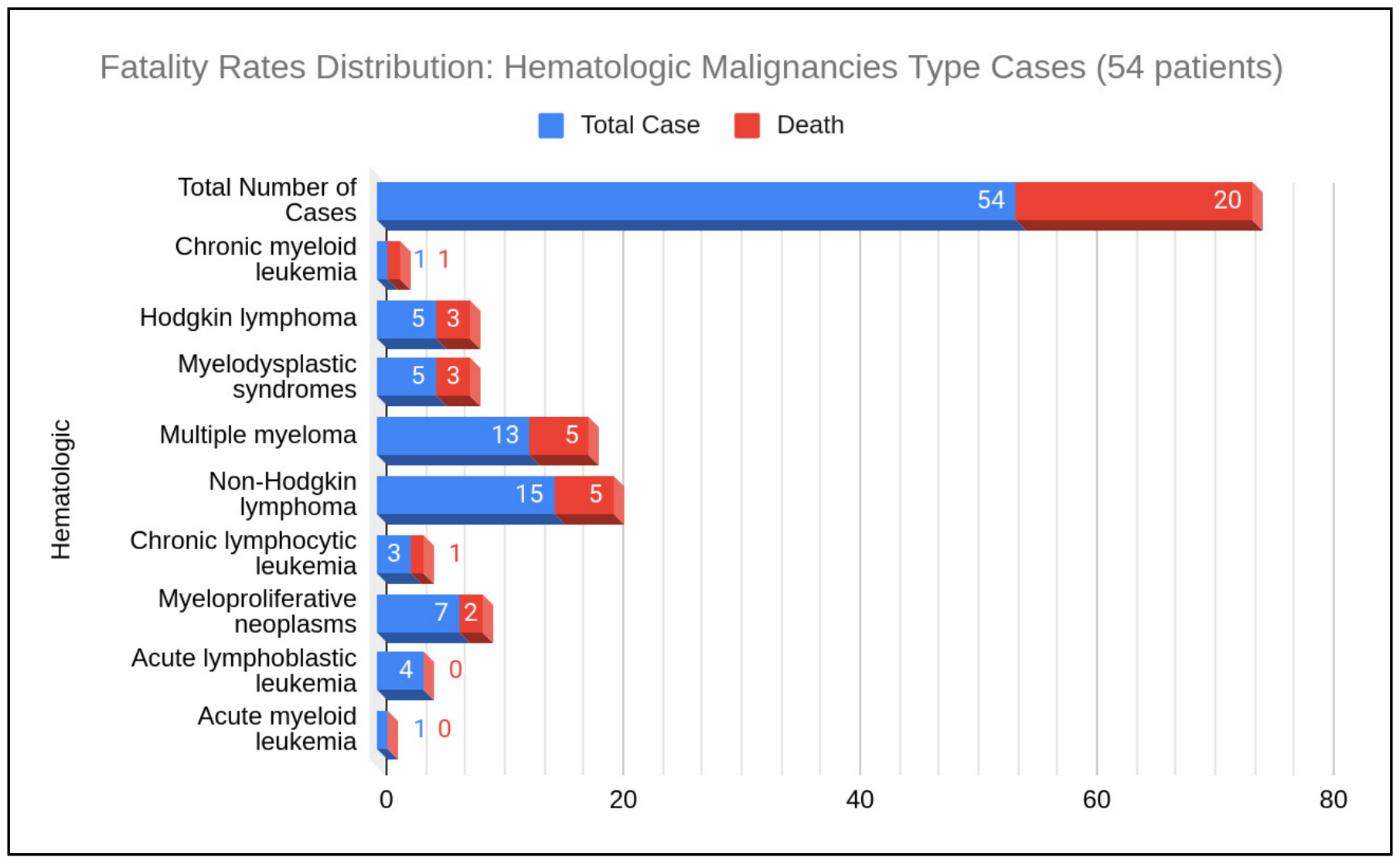

Figure 10. Fatality distribution amongst the Hematologic Malignancies cancer patient with COVID-19

It was found that death rates in cancer patients with COVID-19 were significantly increased in all age groups, but older age was correlated with greater fatality. The age-distribution was such that most of the patients were adults (98.6\%), ranging from 10 to 92 years and their median age was 69 years and 58\% were male population.

\section{Cancer management in Italy [18]}

Italy has observed the second largest number of deaths in Europe after the United Kingdom with around 34000 deaths and counting as on June 12, 2020 [19]. The urgency to develop, and embrace an exceptional strategy to restrict viral infection by the universal Italian national health system is justified by the current fatality upshot which is $14 \%$ of the total cases.

Also, it was found that especially older people undergoing cancer treatment have a higher chance of being critically affected by COVID-19. The data reveals the deceased patients were of median age 80 years and often $20 \%$ had a cancer diagnosis. As of now, the vital data (such as cancer type, stage, and status of ongoing therapy) on deceased cancer patients due to infection are lacking.

Given the aforementioned outline, the initial step was taken toward renewing the Elderly Cancer Care and aging-related problems by numerous organizations such as the Italian Group for Geriatric Oncology (GIOGER). It is well acknowledged that older patients with cancer are at twofold risk because of age and immunosuppressive therapy. The country faces emergencies not only w.r.t healthcare but also in socio-economic ethical aspects. Thus, more resources were designated for medical and ICUs, continuous modernization of care programs to lessen the risk of coronavirus pneumonia in elderly cancer patients while restraining the transmission of infected incidents among patients.

In general, the compulsory measures have been taken including the monitoring of body temperature before the entrance of hospital premises; avoiding crowded places; washing hands as per WHO protocol; bypassing any contact with acquaintances with COVID-19 symptoms including marked endemic zones; following social distancing norm of $6 \mathrm{ft}$ or more; wearing PPE for hospital appointments and therapies and educating the cancer patients through personalized phone calls and social media platforms. Avoid overcrowding in waiting rooms, in the chemotherapy area, and parking areas and to guarantee safe social distancing. 


\section{Several specific criteria for cancer patients are:}

1. Reduction of immunodeficiency \& iatrogenic treatment to withdraw any intensification of severe infection.

2. Contemplate postponing the follow-up say, 6-12 months for disease-free aging cancer patients, and instead implement telephonic or telematic triage.

3. Whereas for patients with active disease, it is prudent to evaluate clinical requirements by assessing laboratory tests and should be placed on oral medication.

4. To limit the turnover of patients, families, and caregivers, the hospital is expanding telemedicine and presence of a single companion for a limited time for inpatients

5. Continuation of antineoplastic medications and regular assessment on a case by case basis and according to the Italian Drug Agency (AIFA), the drug refill time is reasonable to extend.

6. The intravenous regimen with a more extended interval say., 4 weeks should be exercised whereas less frequent once can be avoided

7. Prophylactic use of growth agents at high risk of myelosuppression is recommended for several lengthening cycles of chemotherapy

8. Rather than IV-intravenous assistants (chemotherapy or checkpoint inhibitors) prefer Oral targeted treatment. Also, perioperative chemotherapy with a greater chance of neutropenia which should be withdrawn, resectable cancer patients.

Guideline for the benefit of its staff, patients, and families by American University of Beirut Medical Center (AUBMC), Lebanon [20] [21]

The current crisis demands specific readiness response and combined efforts besides the common rules which existed earlier for the well-being of community health. So, to begin with, assessment of the immunity has to be extended to also cover coronavirus infection for all the healthcare members while being examined by the nurses. Next up, the safety of patients is of the highest precedence for which AUBMC proposed discrete measures such as:

1. All visitor to be screened before entering the hospital

2. Mandatory Face masks for everyone

3. Physical distancing in waiting areas, queue, and public

4. Illustrating stickers to assure a $2 \mathrm{mtr}$ distance amongst all visitors

5. Plexiglass at the emergency division (ED) patient, desks \& clinics

6. Regular and stringent sanitizing and housekeeping measures

7. Scheduling patient visits to avoid congestion in waiting areas

8. No visiting constraint yet

9. Digital signage with visual warnings and instructions at entrances

10. Guides for Self-care \& self-isolation to stay at home patients

Additionally, AUBMC has directions in various aspects related to cancer patients as shown in Figure 11:

1. Pandemic Evaluation Clinic and Center, a separate area retrofitted with global COVID-19 specifications for ED-Emergency Department patients with COVID-19 symptoms

2. No passage to the central buildings to symptomatic or tested positive COVID-19 patients. Further, for assured tracking obligations, a dedicated unit of specialists for any positive cases

3. Rigorously staffing policies to guarantee that the crucial facilities are accessible as required. Infected hospital representatives to self-quarantine at home \& report if any alerting indications

4. Home Lab Collection for blood samples and tests

5. Tele-Health an online video consulting with doctors

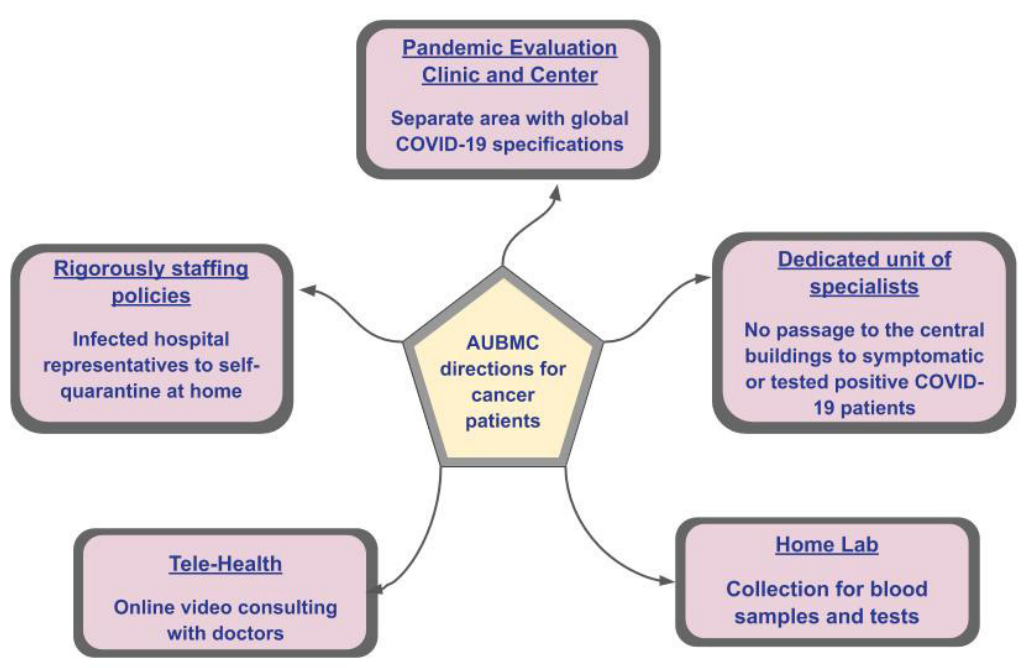

Figure 11. AUBMC's directions in various aspects related to cancer patients 
List concerning particular Dos and Don'ts for Medical Staff:

1. Washing hands with soap \& water for a minimum of 20 seconds

2. Use of sanitizers between examinations of patients

3. No touching of nose/lips/eyes

4. No handshaking

5. Maintaining a physical distance of 6 to $8 \mathrm{ft}$ in waiting and examination rooms

6. Wearing of masks properly

7. No touching and altering masks to avoid the unconscious spread of the virus

8. Correct disposal and removal of masks

9. Usage of gloves while examining patients

10. Avoid crowds, public transportation, cafes, restaurants, unnecessary travel, \& people who are ill

11. Stay home as much as feasible

12. Be well-nourished

13. No smoking of cigarettes or use hookah etc.

14. Shop for essential items at times when there are no crowds or have a family member or friend do it for you if you are ill or in a high-risk group

15. Disinfecting commonly touched ítems

\section{The European Society for Medical Oncology (ESMO) [22]}

European Society for Medical Oncology, being the foremost expert organization for medical oncology has introduced an exhaustive set of guidelines on the management of cancer patients during the COVID-19 pandemic. At a high level, the guidance works by a grouping mechanism i.e. by classifying patients who may be at particular risk concerning COVID-19 and prioritizing them. Additionally, it provides a list of symptoms, precautions, and actions that should be taken in the event of any such infectious indications. This is to be used to alleviate the negative effects of the ongoing coronavirus on the supervision of cancer patients.

The ESMO advises norms with high, medium, and low priorities for each type of cancer such as Brain Tumor, Breast Cancer, Cervical Cancer, Colorectal Cancer, Endometrial Cancer, Epithelial Ovarian Cancer, GastroOesophageal Tumour, Hepatocellular Carcinoma, Hodgkin Lymphoma, Indolent Lymphoma, Lung Cancer, Melanoma, Multiple Myeloma, Pancreatic Cancer, Prostate Cancer, Sarcoma, and Urothelial Bladder Cancer.

\section{Explicit risky cases}

The cancer patients who fall into particular categories of risk concern are represented in Figure 12 includes patient currently getting chemotherapy, or received one within the last three months; undergoing extensive radiotherapy; transplantations of bone marrow or stem cell in the last six months; taking immunosuppressive drugs, and having any type of blood or lymphatic system cancer which leads to weakening of the immune system, also for conditions such as chronic leukemia, lymphoma, or myeloma.

The cancer patients with a weakened immune system also include Leukocytopaenia, Low immunoglobulin levels, and long lasting immunosuppression (steroids, antibodies).

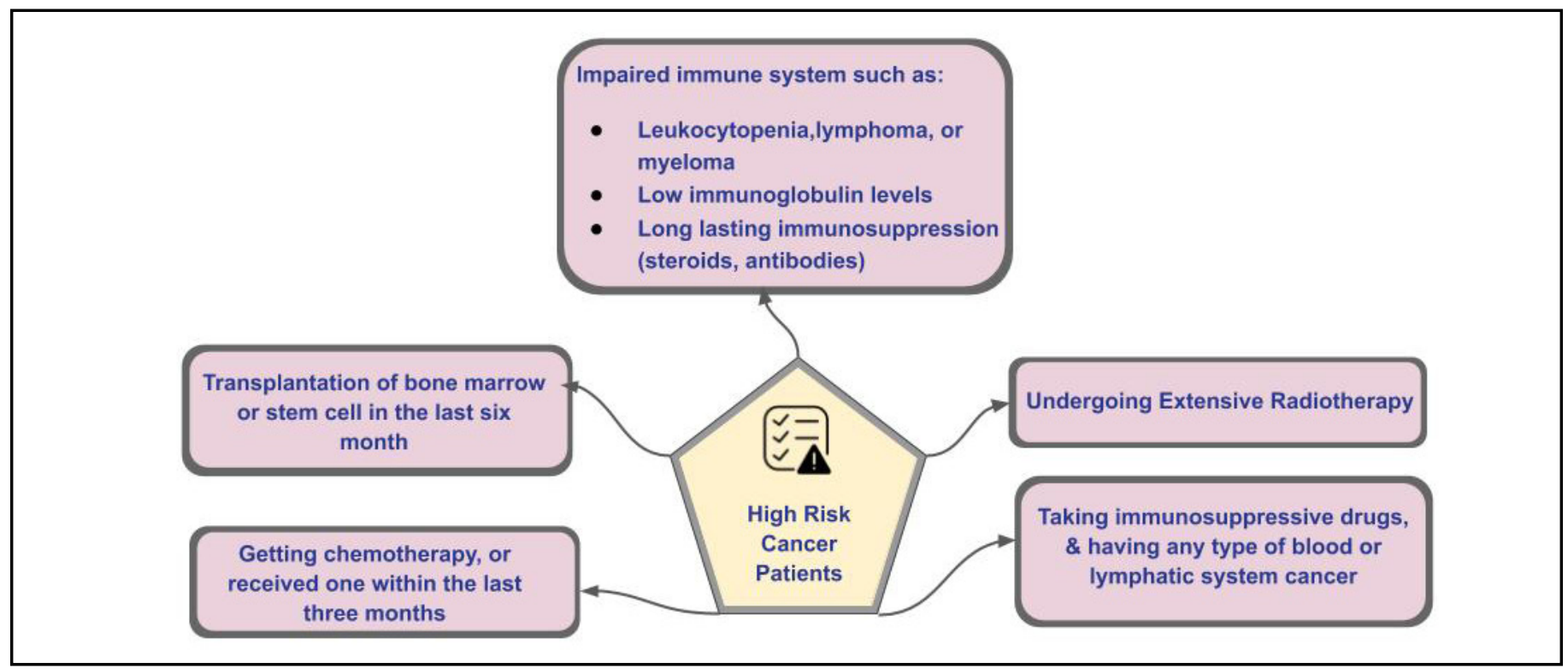

Figure 12. Category of the high-risk Cancer patient as layout by ESMO 


\section{Effect of COVID-19 pandemic on the cancer care}

If a cancer patient develops symptoms of COVID-19, the medical priority will be to isolate the patient and treat the COVID-19 infection rather than cancer. The cancer treatment will resume once the cancer patient has recovered sufficiently from COVID-19. Also, attending the hospital during the COVID-19 pandemic can put the cancer patient at risk of infection. Therefore, to keep the patient safe the cancer care system needed to adapt while the pandemic is ongoing. The aim is to reduce the time spent in a hospital setting whilst still providing the care that is needed.

To minimize face-to-face contact, telephone or video consultations, particularly for pre-treatment meetings and follow-up appointments are recommended. The blood tests usually done at the hospital may be relocated to a local center instead. Medication may be delivered to the patient's home, or a drive-through pick-up point may be arranged. Repeat or electronic prescription periods are also being extended in many areas to allow patients to access their home-based cancer treatment (e.g. oral treatments) without the need for additional prescribing appointments during the pandemic.

\section{Recommended treatment decision}

ESMO actively prescribes remote contact, similar to Telehealth where the distribution of health-related services and information is via electronic information and telecommunication technologies such as phone or webbased communication between patients and doctors. The decision is based on the risk-benefit profiles of cancer treatment to be analyzed in the context of the ongoing pandemic. According to ESMO, "The decisions for treatment initiation or continuation must be discussed for both uninfected patients and SARS-CoV2-positive patients if they are a- or paucisymptomatic, still fit to be treated and willing to do so after proper risk/benefit explanation".

The complete set of benefits and risks of present cancer therapy in the setting of the COVID-19 pandemic is given in Figure 13.

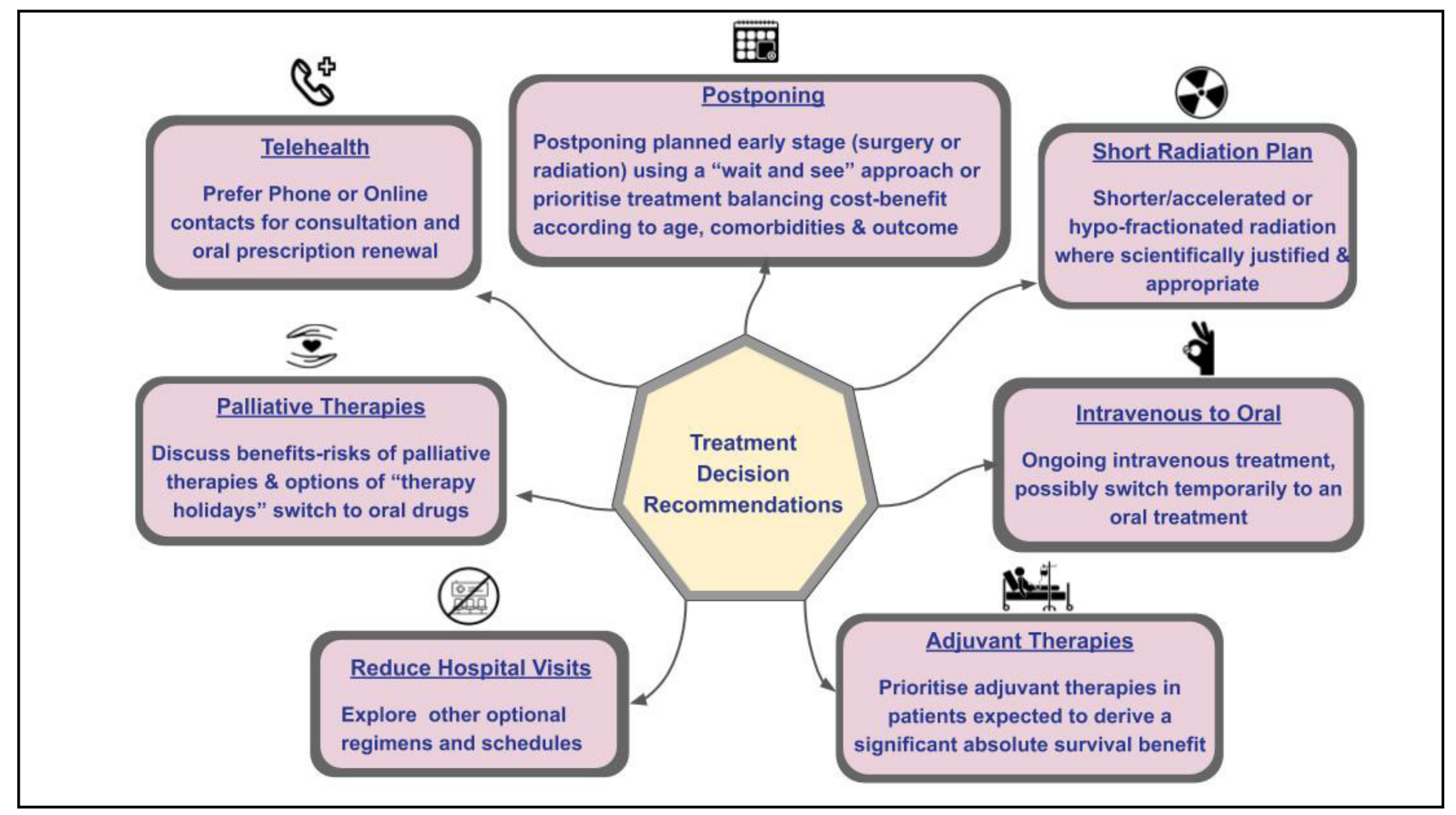

Figure 13. Protocol for cancer patient with a chance of Radiologic manifested Pneumonia and Chemotherapy-induced Neutropenia 


\section{Cancer patient with fever}

Especially, for cancer patients with fever are prescribed to follow the guideline as shown in Figure 14 in order to make sure that it does not spread amongst other cancer patients in an oncology center, inspection is outside the high concentration area, any possibility of coronavirus should be evaluated and oral antibiotic therapy is preferred.

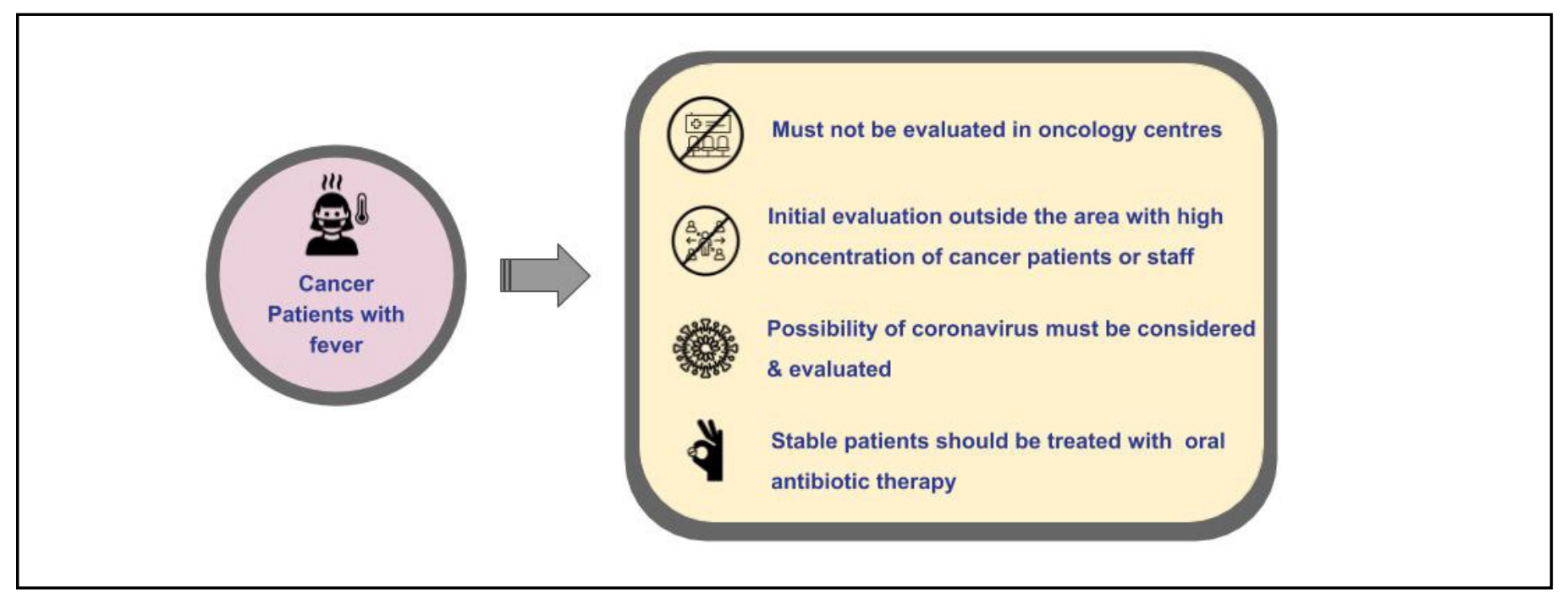

Figure 14: Protocol for Cancer Patient with fever

\section{Prioritization of cancer patient}

For prioritization, the ESMO has composed three levels of urgency for cancer patients i.e. Tier 1 as a high priority, Tier 2 as a medium priority, and Tier 3 as low priority Intervention. The criteria are based on the value-based prioritization and clinical cogency of the interventions is described in Figure 15.

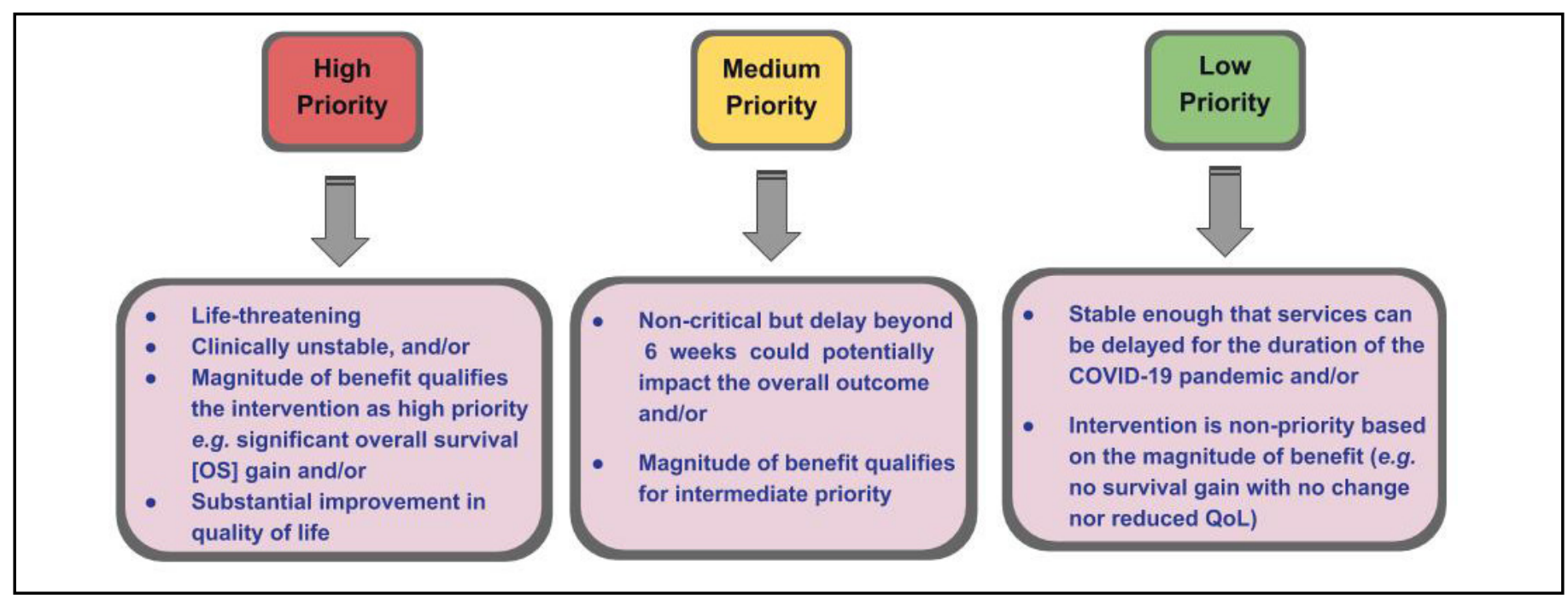

Figure 15. Cancer patient prioritization based on associated risk-benefit

\section{Gynecological cancer patient [23]}

In the recently published article on the Indian Journal of Gynecologic Oncology [23], it is suggested that gynecologic oncologists must follow a principled, conflict-sensitive "do no harm" strategy and extend providing evenhanded, compassionate, and safe care to cancer patients. Besides accessing the current rise in the number of COVID-19 cases in India the patients are advised to avoid visiting the hospital unless there is an emergency or very stringent and definite outline of management as shown in Figure 16. 
The subsequent suggestions have to be examined if the COVID crisis continues:

1. Diagnostic evaluation in 4 weeks from the initial stage for women with suspected cervical cancer is perfect or schedule diagnostic conization/LEEP(loop electrosurgical excision) after careful counseling and evaluation

2. Screening can defer up to 3 months for high-grade lesions on cytology and counseling \& follow until the crisis is resolved ( i.e. 6-12 months) for low-grade lesions patients.

3. Re-evaluated operability for women with early cervical cancer waiting for the last 6-8 weeks \& surgery is preferred if resources permit; otherwise, Neoadjuvant chemotherapy (NACT) with ongoing crises beyond 8 weeks.

4. Consider a non-surgical option for women with lowrisk uterine cancer with expected delay beyond 6-8 weeks otherwise careful counseling \& continuation with NACT for women with ovarian cancer can complete all 6 cycles but if resumed then prioritize surgical options.

5. Consider neoadjuvant chemoradiation for rare cancers like vulva and vagina.

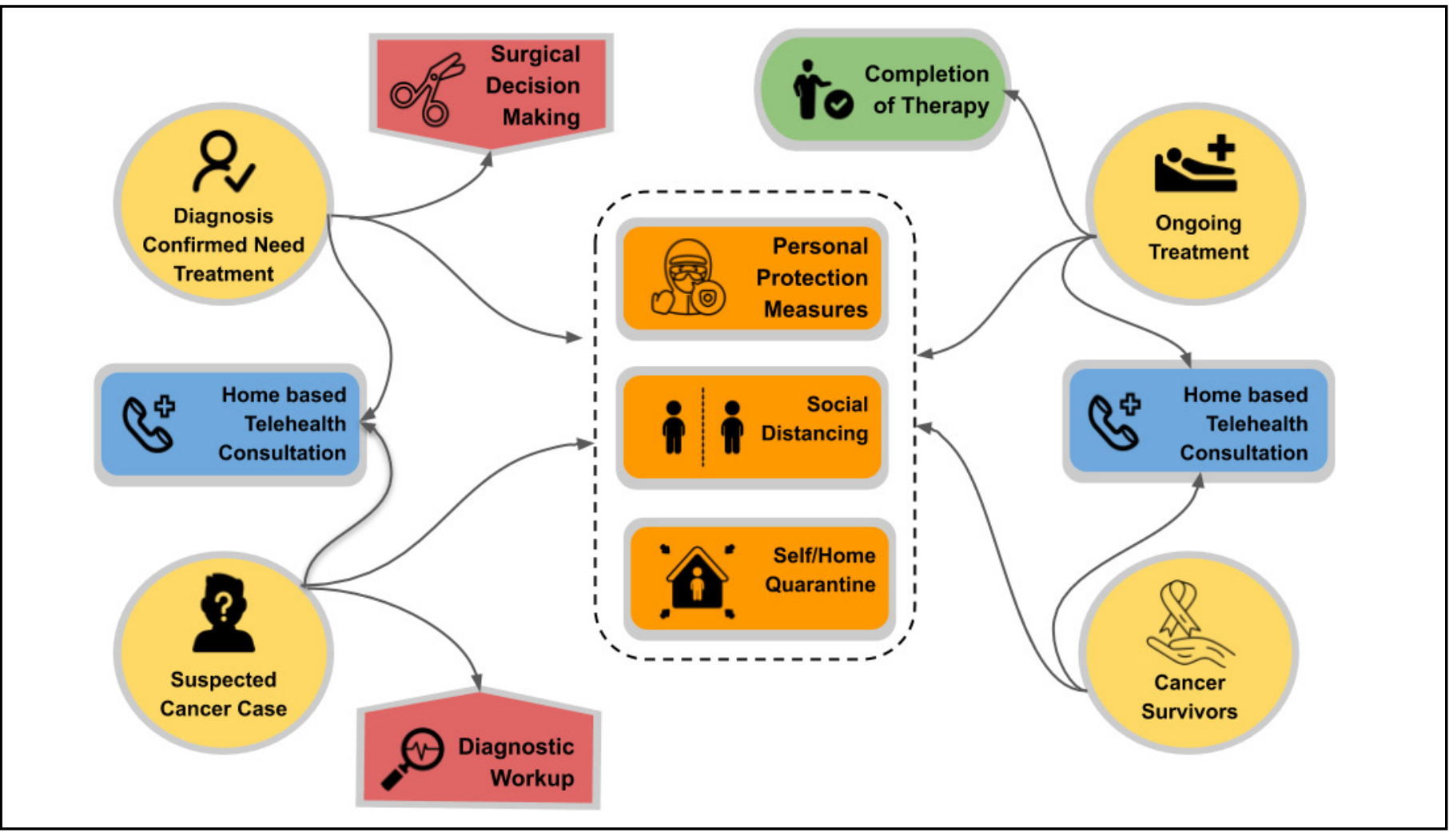

Figure 16. Suggested algorithm for the management of women with gynecological cancers during COVID-19 epidemic

\section{Overall considerations}

Overall, it is time for us to introspect and consider being a part of managing this unexpected pandemic situation and confront the aforementioned catastrophe as a unit exercising one step at a time and understanding the responsibility at an individual level. And to do so we must withdraw from any unfruitful participation and make sure we reduce or avoid the need for selective operations or surgeries. The clinicians and staff who are on the frontline may require to adapt more quickly to various territories more than their routine. But they need to be supported by the general public.
Moreover, bypass any prolonged operation without consultation; perhaps a 7-day work schedule needs to be embraced; suspend prolonged follow-up and use more conventional Tele-health or virtual clinics. As far as CT scans are considered then it may be confined because it will enhance the chances of infection.

\section{Summary}

In general cancer patients have compromised immune systems due to chemo, and other therapeutics. In extension to following all the pandemic guidelines, they can enhance their immune system by having a healthy sleep schedule, withdraw smoking, avoid food that causes inflammation, such as easy sugars in cookies, cakes, 
candies, and soda rather consume more bounteous fruits and vegetables and exercising at or around the home, such as taking a light walk, cardio, etc. [24]

COVID-19 could be a grave concern for cancer patients, but by taking proper precautions and obeying the required guidelines, they can protect themselves and others. The pandemic will alter the broader oncology community in the future too, with the dissolution of conferences and reduced work on research, education, etc. [25]

This special American Association for Cancer Research (AACR) colloquium will concentrate on the presentation of emerging data in basic, clinical, and epidemiologic research related to COVID-19 and cancer [26]. COVID-19 has remodeled our approaches to caring for patients with cancer, and the negative ramifications related to cancer outcomes and psychosocial disorders and methods to mitigate these risks will be addressed [27]. The amplified incidence of critical COVID-19 in certain ethnic backgrounds and association with socioeconomic disparities will be highlighted. COVID-19 has revitalized innovation by cancer investigators to address this pandemic, including new diagnostic assays using nucleotide sequencing and serologic methods that were previously developed to study cancer; the development of new virus-targeted drugs based on the knowledge of COVID-19 proteins as has been done for years with targeted therapies for cancer; the testing of nextgeneration vaccines with similar principles as cancer vaccines; and the use of repurposed oncology drugs to treat COVID-19 patients. Despite the negative impacts of COVID-19 upon society and patients with cancer, the pandemic has hastened innovative changes to cancer care that are transforming patient access to treatments and clinical trials. Advances such as the implementation of telehealth, remote consenting for clinical trials, and access to home infusions, among others, will be discussed. These changes in the conduct of clinical trials have regulatory implications, and regulators, investigators, and industry specialists will discuss their impact on drug development.

\section{Conflict of interest}

The authors declare no conflict of interest.

\section{References}

1. "Cancer Patients \& COVID-19 - What You Need to Know and How to Reduce Your Risks". news. cancerconnect.com. May 2020. https://news.cancerconnect.com/treatment-care/cancer-patientscovid-19-what-you-need-to-know-and-how-toreduce-your-risks-E-1SV5d9mk6w6hphSlU--g

2. "Common Questions about the New Coronavirus
Outbreak”. March 2020. https://www.cancer.org/ latest-news/common-questions-about-the-newcoronavirus-outbreak.html

3. "Why People with Cancer are More Likely to Get Infections". March 2020. https://www.cancer.org/ treatment/treatments-and-side-effects/physicalside-effects/low-blood-counts/infections/whypeople-with-cancer-are-at-risk.html

4. "COVID-19 Patient Care Information". ASCO, Mar. 18, 2020. https://www.asco.org/asco-coronavirus-information/care-individuals-cancer-during-covid-19

5. ESMO, "ESMO management and treatment adapted recommendations in the COVID-19 era: Lung cancer." March 2020. https://www.esmo.org/ guidelines/cancer-patient-management-duringthe-covid-19-pandemic/lung-cancer-in-the-covid-19-era

6. ESMO, "Cancer Care During the COVID-19 Pandemic: An ESMO Guide for Patients." May 2020. https://www.esmo.org/for-patients/patientguides/cancer-care-during-the-covid-19-pandemic

7. V. Mehta et al., "Case Fatality Rate of Cancer Patients with COVID-19 in a New York Hospital System," Cancer Discov., May 2020, doi: 10.1158/2159-8290.CD-20-0516.

8. "Guidelines for Cancer Patients during Corona Virus Pandemic," Tamil Nadu News, Chennai News, Tamil Cinema News, Tamil News, Tamil Movie News, Power Shutdown in Chennai, Petrol and Diesel Rate in Chennai, March 23, 2020. https://chennaivision.com/guidelines-for-cancerpatients-during-corona-virus-pandemic/

9. Anita Ramesh, "Awareness Programme COVID-19 and Cancer Patient. March 20202. https:// www.youtube.com/watch? $v=$ TywlcFQoKoo

10. Anita Ramesh, "People with chronic diseases at higher risk of infection." The Hindu, Mar. 25, 2020 .

11. C. S. Pramesh and R. A. Badwe, "Cancer Management in India during Covid-19," N. Engl. J. Med., vol. 382, no. 20, p. e61, May 2020, doi: 10.1056/ NEJMc2011595. 
12. H.-Y. Zhang et al., "A Multicentre Study of 2019 Novel Coronavirus Disease Outcomes of Cancer Patients in Wuhan, China," medRxiv, p. 2020.03.21.20037127, Apr. 2020, doi: 10.1101/2020.03.21.20037127.

13. H. Mei, X. Dong, Y. Wang, L. Tang, and Y. Hu, "Managing patients with cancer during the COVID-19 pandemic: frontline experience from $\mathrm{Wu}-$ han," Lancet Oncol., vol. 21, no. 5, pp. 634-636, May 2020, doi: 10.1016/S1470-2045(20)30238-2.

14. "Risk for COVID-19 may be higher among those with cancer, Wuhan data suggest." March 2020. https://www.healio.com/news/hematology-oncology/20200326/risk-for-covid19-may-be-higher-among-those-with-cancer-wuhan-data-suggest

15. J. Yu, W. Ouyang, M. L. K. Chua, and C. Xie, "SARS-CoV-2 Transmission in Patients With Cancer at a Tertiary Care Hospital in Wuhan, China," JAMA Oncol., Mar. 2020, doi: 10.1001/jamaoncol.2020.0980.

16. "COVID-19: Data Summary - NYC Health." May 20202. https://www1.nyc.gov/site/doh/covid/covid-19-data.page

17. "Cancer Prevention and Screening - NYC Health." May 2020. https://www1.nyc.gov/site/doh/health/ health-topics/cancer-prevention.page

18. L. Fratino, G. Procopio, M. Di Maio, S. Cinieri, S. Leo, and G. Beretta, "Coronavirus: Older Persons with Cancer in Italy in the COVID-19 Pandemic," Front. Oncol., vol. 10, April 2020, doi: 10.3389/ fonc. 2020.00648 .

19. "Italy Coronavirus: 238,011 Cases and 34,561 Deaths - Worldometer." June 2020. https://www. worldometers.info/coronavirus/country/italy/

20. "AUBMC Adopts Safety Measures for the Welfare of its Community," AUBMC. May 2020. http:// aubmc.org.lb:80/Pages/AUBMC-Adopts-SafetyMeasures-for-the-Welfare-of-its-Community. aspx
21. N. Bitar, J. Kattan, H. R. Kourie, D. Mukherji, and N. E. Saghir, "The Lebanese Society of Medical Oncology (LSMO) statement on the care of patients with cancer during the COVID-19 pandemic," Future Oncol., vol. 16, no. 11, pp. 615-617, Apr. 2020, doi: 10.2217/fon-2020-0252.

22. ESMO, "Cancer Patient Management during the COVID-19 Pandemic." March 2020. https://www. esmo.org/guidelines/cancer-patient-management-during-the-covid-19-pandemic

23. N. Bhatla and S. Singhal, "The COVID-19 Pandemic and Implications for Gynaecologic Cancer Care," Indian J. Gynecol. Oncol., vol. 18, no. 2, April 2020, doi: 10.1007/s40944-020-00395-7.

24. "How Do Cancer Patients Boost Their Immune System During the COVID-19 Pandemic?"' SurvivorNet. March 2020. https://www.survivornet. com/articles/how-do-cancer-patients-boosttheir-immune-system-healthy-during-the-covid19-pandemic/

25. T. L. Oncology, "COVID-19: global consequences for oncology," Lancet Oncol., vol. 21, no. 4, p. 467, Apr. 2020, doi: 10.1016/S1470-2045(20)30175-3.

26. "AACR Virtual Meeting: COVID-19 and Cancer | AACR Meetings," American Association for Cancer Research (AACR). July 2020. https://www.aacr. org/meeting/aacr-virtual-meeting-covid-19-andcancer/

27. J. van de Haar et al., "Caring for patients with cancer in the COVID-19 era," Nat. Med., vol. 26, no. 5, Art. No. 5, May 2020, doi: 10.1038/s41591-0200874-8. 